\title{
Treatment Strategy for Recurrent Multiple Hepatocellular Carcinoma*
}

\author{
Toru Ishikawa
}

Department of Gastroenterology and Hepatology, Saiseikai Niigata Daini Hospital, Niigata, Japan.

Email: toruishi@ngt.saiseikai.or.jp

Received January $2^{\text {nd }}, 2013$; revised February $1^{\text {st }}, 2013$; accepted February $10^{\text {th }}, 2013$

\begin{abstract}
Hepatocellular carcinoma (HCC) has some unique characteristics in the digestive organ cancer. Therefore it is very important to select an appropriate treatment for recurrent HCC according to several situations such as the type of recurrence, previous treatments and conditions of patient. This article is a review of the concept of recurrent multiple HCC and its therapeutic strategies.
\end{abstract}

Keywords: Hepatocellular Carcinoma; Multiple Recurrence; Strategy

\section{Introduction}

Hepatocellular carcinoma (HCC) has the following unique characteristics: limitations of surgery due to complicating liver cirrhosis in many cases; and a 5-year recurrence rate of approximately $80 \%$ after radical treatment, which is much higher than that of other gastrointestinal carcinomas because of its nature as multicentric carcinogenesis secondary to chronic liver disease or intrahepatic metastasis $[1,2]$. It would be ideal to radically treat multiple HCC with surgery, but in fact, it is primarily treated with medical therapy due to problems such as residual hepatic function and general status. This article is a review of the concept of recurrent multiple HCC and its therapeutic strategies.

\section{Concept of Recurrent Multiple Hepatocellular Carcinoma}

According to the report on the 18th nationwide follow-up survey of primary liver cancer (2004-2005) by the Liver Cancer Study Group of Japan, 57.7\%, 17.3\%, 7.9\%, 3.2\%, $1.5 \%$, and $12.4 \%$ of 18,255 cases had $1,2,3,4,5$, and 6 or more lesions, respectively, showing that nearly half of the cases were multiple HCC [3]. In addition, since many patients with a single lesion experience recurrence, the prognosis of HCC is determined by the therapeutic strategy for multiple HCC. Intrahepatic multiple lesions are attributable to multicentric carcinogenesis or intrahepatic metastasis from the primary lesion, and the etiology has a

\footnotetext{
*No conflicts of interest in the manuscript.

The authors declare that they do not have any current financial arrangements or affiliations with any organization that may have a direct interest in their work.
}

significant impact on treatment selection and prognosis prediction.

Multicentric carcinogenesis is characterized by multiple HCC lesions and may involve the following lesions, which are estimated to occur and grow in situ:

1) Adenomatous hyperplasia or early HCC with the preexisting liver structure intact;

2) HCC with moderately or poorly differentiated cancer tissue surrounded by well-differentiated cancer tissue.

On the other hand, intrahepatic metastasis may involve the following lesions:

1) Portal vein tumor thrombus or cancer lesion originating from portal vein tumor thrombus;

2) Cancer lesions that are distributed in inverse proportion to the distance from the largest tumor;

3) Cancer lesion that is isolated but located near the largest tumor, and definitely smaller and equally or less differentiated than the largest tumor.

In the clinical setting, however, it is often difficult to make a differential diagnosis between primary cancer (multicentric carcinogenesis) and metastatic cancer (intrahepatic metastasis). In Japan, multicentric carcinogenesis should first be considered, because many cases of HCC result from chronic inflammation due to hepatitis B virus (HBV) or hepatitis $\mathrm{C}$ virus (HCV) infection.

\section{Treatment Strategy}

\subsection{Treatment Strategy Prior to Recurrent Multiple Hepatocellular Carcinoma to Maintain Residual Hepatic Function}

To prevent recurrence of HCC secondary to chronic viral 
hepatitis after radical treatment, interferon IFN therapy may be useful in inhibiting multicentric carcinogenesis, whereas chemotherapy based on transcatheter hepatic arterial infusion (TAI) is recommended to inhibit intrahepatic metastasis.

We evaluated the treatment response and hepatic functional reserve in patients who received combination therapy with PEG-IFN $\alpha$-2b and ribavirin (RBV) after radical treatment of HCV-related HCC [4]. Fifty-four patients with primary HCV-related HCC (stage I/II) were included in the study, and the survival rate, metachronous recurrence rate, and hepatic functional reserve were assessed. Of these patients, 29 patients received combination therapy with PEG-IFN $\alpha$-2b and RBV after treatment of HCC (IFN secondary treatment group), and the other 25 patients did not (non-secondary treatment group). The 1-year and 3-year cumulative survival rates were $100 \%$ and $90.2 \%$ in the IFN secondary treatment group, and $96.0 \%$ and $61.2 \%$ in the non-secondary treatment group, respectively, showing a significant difference between the groups. Multivariate analysis identified secondary IFN treatment as a significant factor. In the PEG-IFN $\alpha$-2b/RBV group, serum albumin levels transiently decreased, but increased later, indicating improvement in hepatic functional reserve [2] (Figure 1). These results show that combination therapy with PEG-IFN $\alpha$ $2 \mathrm{~b}$ and RBV following treatment of HCC contributes to improvement in hepatic functional reserve, and increases treatment options in case of recurrence.

\subsection{Treatment Strategy for Multicentric Carcinogenesis}

Treatment of HCC should be selected according to the degree of progression (number and size of tumors, presence or absence of tumor embolus, etc.) $[5,6]$ and severity of hepatic impairment. In addition, the treatment strategy for multiple HCC differs depending on whether it is

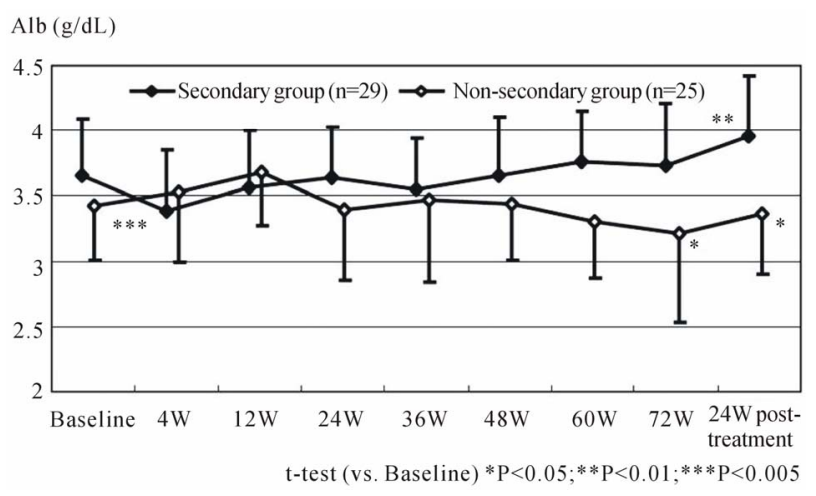

Figure 1. Serial changes in mean albumin levels compared between secondary IFN and non secondary IFN group (Ref 4; T. Ishikawa et al. Hepatogastroenterology. 2012 Mar-Apr; 59(114): 529-532). multicentric occurrence or intrahepatic metastasis [7]. For multicentric occurrence, effective local treatment should be selected for cure, because individual lesions are localized. Once IFN therapy has improved hepatic functional reserve, HCV-related HCC should be treated more radically. We have obtained preliminary data demonstrating that transcatheter arterial chemoembolization (TACE) combined with radiofrequency ablation (RFA) for mass reduction improves the prognosis of multiple recurrent HCC, even in cases that are not within the Milan criteria (Figure 2). Therefore, the treatment strategy for multicentric occurrence should be aimed at cure, for instance, by mass reduction, even for highly advanced, recurrent cancer to convert it to resectable disease wherever possible, as in chemotherapy for colorectal cancer.

\subsection{Treatment Strategies for Intrahepatic Metastasis}

For intrahepatic metastasis, on the other hand, it is essential to select treatment not only for overt lesions, but also for micrometastasis, and combination therapy including intra-arterial treatment such as TACE is required for initial or recurrence treatment.

In the clinical setting, it is general consensus that the presence of up to three lesions is probably associated with multicentric occurrence, for which local necrosis therapy such as resection or radiofrequency ablation is indicated, whereas the presence of at least four lesions is probably associated with intrahepatic metastasis, for which TACE is indicated [5,6].

\subsection{Treatment Strategy and Problems with TACE}

In Japan, TACE, which is recommended for inoperable patients for whom local puncture therapy is not indicated according to the guidelines for the management of liver cancer, plays a central role in the treatment of recurrent advanced multiple HCC $[5,6]$. TACE is primarily per-

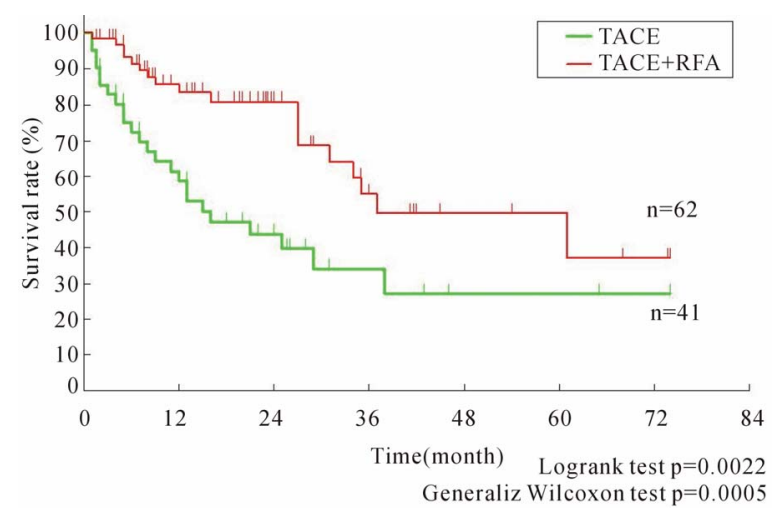

Figure 2. Kaplan-Meier survival curve for receiving TACE with/without RFA for advanced HCC. 
formed as chemolipiodolization, using anticancer drugs mixed with lipiodol $[8,9]$. There have been several retrospective reports of TACE with anthracyclines versus platinum compounds, suggesting the usefulness of platinum compounds $[10,11]$. However, no effective drug has been identified, because no prospective comparative studies have been reported, and various anticancer drugs, including epirubicin, cisplatin, mitomycin $\mathrm{C}$, and doxorubicin, were used with intercenter variance. Results from ongoing Phase III studies (Epirubicin vs Cisplatin, Epirubicin vs Miriplatin) are awaited [12]. Ikeda et al. reported that the 1-year and 2-year survival rates were $89.9 \%$ and $75 \%$ in 99 patients with unresectable HCC, respectively, in a multicenter Phase II study of TACE with epirubicin [13]. According to a nationwide survey reported by Yamakado et al., on the other hand, the 1-year and 3-year survival rates were $92 \%$ and $62.9 \%$ in 815 patients who underwent TACE with epirubicin in 43 institutions in 20032004, respectively [14]. Survival of patients who could undergo selective TACE was significantly longer than that of those who could not. In this report, 651 (79.9\%) of the 815 patients had recurrence: local recurrence in $42.2 \%$ (275/651) and intrahepatic distant tumor recurrence in $57.3 \%$ (373/651). Osuga et al. reported the results from a multicenter study of lipiodol-free TACE with gelatin sponge particles following TAI with DDP-H (IAcall, CDDP powder for arterial infusion) in bilobar multiple advanced HCC [15]. Disease had advanced after previous TACE with epirubicin in $59 \%$ of patients. The 1-year and 2-year survival rates were $75 \%$ and $51.3 \%$, respectively, and the RECIST and EASL response rates were $25.6 \%$ and $65.2 \%$, respectively, showing that whole liver arterial infusion and embolization could be performed safely and effectively in bilobar multiple advanced HCC.

In recent years, introduction of selective TACE has contributed to improvement in the outcome of TACE with better local control. On the other hand, it is imperative to establish treatment strategies for post-TACE multiple recurrence, especially for those with a high incidence of intrahepatic distant tumor recurrence.

We therefore reviewed the usefulness of platinumcontaining drugs, which may be key drugs in the treatment of HCC.

\section{Significance of Platinum-Containing Drugs in Transhepatic Arterial Infusion}

After treatment of HCC, the remaining liver is still in precarcinogenesis. The protective effect of chemotherapy on the remaining liver against recurrence may be demonstrated if it is effective in patients with a high probability of intrahepatic metastasis, which is associated with a high recurrence rate. While intra-arterial chemotherapy is not highly appreciated in the West, hepatic arterial infusion resulting in high local drug concentrations is expected to improve the prognosis and prevent recurrence, because lesions are often localized to the liver even in the advanced stage of HCC, and various regimens have been attempted without reaching consensus on the administration method or dose level. We evaluated the effectiveness of additional chemotherapy of the platinum-containing drugs carboplatin (CBDCA) and DDP-H in preventing intrahepatic distant tumor recurrence [16]. A total of 78 patients with a diagnosis of primary stage I/II HCC who underwent TACE and RFA after whole liver arterial infusion of CBDCA (25 patients) or DDP-H (53 patients) for local control and recurrence prevention were followed up on a long-term basis, and the clinical background factors, intrahepatic distant tumor recurrence rate, and intrahepatic distant tumor recurrence factors were compared between the CBDCA and DDP-H groups. No significant differences were observed in the clinical background factors between the two groups, but the intrahepatic distant tumor recurrence rate was significantly lower in the DDP-H group [3] (Figure 3). In multivariate analysis using Cox's proportional-hazards model, whole liver arterial infusion of DDP-H was identified as an independent factor for privention of recurrence. That is, whole liver arterial infusion of DDP-H significantly priventeded intrahepatic distant tumor recurrence. Significant privention of recurrence by single infusion of DDP-H compared with CBDCA suggests the importance of DDP-Hbased treatment strategy for patients with intrahepatic metastasis.

With no evidence of contribution to survival, there is no mention of TAI in Western guidelines [17]. However, it has been shown that some patients with TACE-refractory HCC were responsive to repeated TAI, and survival was prolonged in these responsive patients. In the current treatment of advanced HCC, hepatectomy, radiofrequency ablation, and TAI, which is the last option available to patients for whom TACE is not indicated or effective, are commonly selected. According to the 18th nationwide follow-up survey of primary liver cancer [1], 85.8\% of 1862 HCC patients treated with chemotherapy

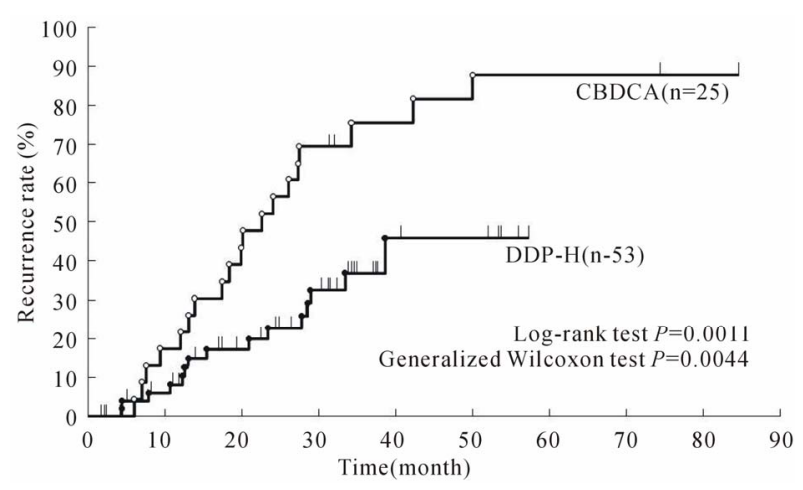

Figure 3. Cumulative intrahepatic distant recurrence rates of CBDCA and DDP-H patients. (Ref 16; T. Ishikawa et al. Cancer. 2011 Sep 1; 117(17): 4018-4025). 
underwent TAI. Since TAI is relatively often selected as the final choice for advanced HCC including recurrent HCC, it is significant to improve the response rate of TAI.

It is necessary to establish treatment strategies with DDP-H-based TAI by conducting prospective randomized control trial $[18,19]$.

\section{Conclusions}

In this article, medical treatment principles for recurrent multiple HCC were reviewed.

In treatment strategies for recurrent multiple HCC, it is very important to select an appropriate treatment according to the degree of progression, and to determine the patient's hepatic functional reserve. It is also important to take the type of recurrence and previous treatments into consideration. On the other hand, it is imperative to establish preventive strategies against precarcinogenesis associated with multicentric carcinogenesis or intrahepatic metastasis still remaining after cure as early as possible and thereby improve the prognosis.

\section{REFERENCES}

[1] S. Shiina, R. Tateishi, T. Arano, et al., "Radiofrequency Ablation for Hepatocellular Carcinoma: 10-Year Outcome and Prognostic Factors," American Journal of Gastroenterology, Vol. 107, No. 4, 2012, pp. 569-577. doi:10.1038/ajg.2011.425

[2] H. Imamura, Y. Matsuyama, E. Tanaka, et al., "Risk Factors Contributing to Early and Late Phase Intrahepatic Recurrence of Hepatocellular Carcinoma after Hepatectomy,” Journal of Hepatology, Vol. 38, No. 2, 2003, pp. 200-207. doi:10.1016/S0168-8278(02)00360-4

[3] M. Kudo, et al., "Report of the 18th Nationwide Follow-Up Survey of Primary Liver Cancer in Japan,” Kanzo (in Japanese), Vol. 51, No. 8, 2010, pp. 460-484.

[4] T. Ishikawa, K. Higuchi, T. Kubota, et al., "Combination PEG-IFN-2b/Ribavirin Therapy Following Treatment of Hepatitis C Virus-Associated Hepatocellular Carcinoma Is Capable of Improving Hepatic Functional Reserve and Survival," Hepatogastroenterology, Vol. 59, No. 114, 2012, pp. 529-532. doi:10.5754/hge10867

[5] N. Kokudo and M. Makuuchi, "Evidence-Based Clinical Practice Guidelines for Hepatocellular Carcinoma in Japan: The J-HCC Guidelines,” Journal of Gastroenterology, Vol. 44, No. 19, 2009, pp. 119-121. doi:10.1077/s00535-008-2244-z

[6] M. Kudo, N. Izumi, N. Kokudo, et al., HCC Expert Panel of Japan Society of Hepatology, "Management of Hepatocellular Carcinoma in Japan: Consensus-Based Clinical Practice Guidelines Proposed by the Japan Society of Hepatology (JSH) 2010 Updated Version,” Digestive Diseases, Vol. 29, No. 3, 2011, pp. 339-364. doi:10.1159/000327577

[7] S. Togo, K. Tanaka, H. Masui, et al., "Usefulness of Pro- phylactic Transcatheter Arterial Infusion of Anticancer Agents with Lipiodol to Prevent Recurrence of Hepatocellular Carcinoma after Hepatic Resection,” International Surgery, Vol. 90, No. 2, 2005, pp. 103-108.

[8] M. Satake, H. Uchida, Y. Arai, et al., “Transcatheter Arterial Chemoembolization (TACE) with Lipiodol to Treat Hepatocellular Carcinoma: Survey Results from the TACE Study Group of Japan," CardioVascular and Interventional Radiology, Vol. 31, No. 4, 2008, pp. 756-761. doi:10.1007/s00270-007-9255-7

[9] K. Takayasu, S. Arii, I. Ikai, et al., Liver Cancer Study Group of Japan, “Overall Survival after Transarterial Lipiodol Infusion Chemotherapy with or without Embolization for Unresectable Hepatocellular Carcinoma: Propensity Score Analysis,” American Journal of Roentgenology, Vol. 194, No. 3, 2010, pp. 830-837. doi:10.2214/AJR.09.3308

[10] H. Yodono, K. Matsuo and A. Shinohara, "A Retrospective Comparative Study of Epirubicin-Lipiodol Emulsion and Cisplatin-Lipiodol Suspension for Use with Transcatheter Arterial Chemoembolization for Treatment of Hepatocellular Carcinoma," Anticancer Drugs, Vol. 22, No. 3, 2011, pp. 277-282. doi:10.1097/CAD.0b013e328342231d

[11] K. Kasai, A. Ushio, K. Sawara, et al., "Transcatheter Arterial Chemoembolization with a Fine-Powder Formulation of Cisplatin for Hepatocellular Carcinoma," World Journal of Gastroenterology, Vol. 16, No. 27, 2010, pp. 3437-3444. doi:10.3748/wjg.v16.i27.3437

[12] ACE500 Study. https://upload.umin.ac.jp/cgi-open-bin/ctr/ctr.cgi?function =brows\&action=brows\&type $=$ summary \&recptno $=$ R0000 01678\&language $=\mathrm{E}$

[13] M. Ikeda, Y. Arai, et al., "Transcatheter Arterial Chemoembolization with Epirubicin and Lipiodol for Unresectable Hepatocellular Carcinoma-Subgroup Analysis of Japanese Patients in Cooperative Study between Korea and Japan,” Japanese Journal of Radiology, Vol. 27, 2012, pp. 299-304.

[14] K. Yamakado, S. Miyayama, S. Hirota, et al., "Hepatic Arterial Embolization for Unresectable Hepatocellular Carcinomas: Do Technical Factors Affect Prognosis?” Japanese Journal of Interventional Radiology, Vol. 30, No. 7, 2012, pp. 560-566. doi:10.1007/s11604-012-0088-1

[15] K. Osuga, Y. Arai, H. Anai, et al., "Phase I/II Multicenter Study of Transarterial Chemoembolization with a Cisplatin Fine Powder and Porous Gelatin Particles for Unresectable Hepatocellular Carcinoma: Japan Interventional Radiology in Oncology Study Group Study 0401,” Journal of Vascular and Interventional Radiology, Vol. 23, No. 10, 2012, pp. 1278-1285. doi:10.1016/j.jvir.2012.06.028

[16] T. Ishikawa, K. Higuchi, T. Kubota, et al., "Prevention of Intrahepatic Distant Recurrence by Transcatheter Arterial Infusion Chemotherapy with Platinum Agents for Stage I/II Hepatocellular Carcinoma," Cancer, Vol. 117, No. 17, 2011, pp. 4018-4025. doi:10.1002/cncr.25989

[17] J. Bruix and M. Sherman, “American Association for the 
Study of Liver Diseases. Management of Hepatocellular Carcinoma: An Update,” Hepatology, Vol. 53, No. 3, 2011, pp. 1020-1022. doi:10.1002/hep.24199

[18] T. Ishikawa, M. Imai, H. Kamimura, et al., "Improved Survival for Hepatocellular Carcinoma with Portal Vein Tumor Thrombosis Treated by Intra-Arterial Chemotherapy Combining Etoposide, Carboplatin, Epirubicin and Pharmacokinetic Modulating Chemotherapy by 5-FU and
Enteric-Coated Tegafur/Uracil: A Pilot Study,” World Journal of Gastroenterology, Vol. 13, No. 41, 2007, pp. 5465-5470.

[19] T. Ishikawa, "Future Perspectives on the Treatment of Hepatocellular Carcinoma with Cisplatin,” World Journal of Hepatology, Vol. 1, No. 1, 2009, pp. 8-16. doi:10.4254/wjh.v1.i1.8 